\title{
COPD and chronic bronchitis risk of indoor air pollution from solid fuel: a systematic review and meta-analysis
}

\author{
Om P Kurmi, ${ }^{1}$ Sean Semple, ${ }^{2}$ Padam Simkhada, ${ }^{2}$ W Cairns S Smith, ${ }^{2}$ Jon G Ayres ${ }^{1}$
}

- Supplementary figures, tables and references are published online only at http://thx.bmj. com/content/vol65/issue3

${ }^{1}$ Institute of Occupational and Environmental Medicine, School of Population and Health Sciences, University of Birmingham, Birmingham, UK ${ }^{2}$ Division of Applied Health Sciences, University of Aberdeen, Population Health Sciences, School of Medicine, Foresterhill, Aberdeen, UK

\section{Correspondence to}

Mr Om P Kurmi, Institute of Occupational and Environmental Medicine, School of Population and Health Sciences, University of Birmingham, Birmingham B15 2TT, UK; 0.kurmi@bham.ac.uk

Received 4 August 2009 Accepted 11 December 2009

\section{ABSTRACT}

Background Over half the world is exposed daily to the smoke from combustion of solid fuels. Chronic obstructive pulmonary disease (COPD) is one of the main contributors to the global burden of disease and can be caused by biomass smoke exposure. However, studies of biomass exposure and COPD show a wide range of effect sizes. The aim of this systematic review was to quantify the impact of biomass smoke on the development of COPD and define reasons for differences in the reported effect sizes.

Methods A systematic review was conducted of studies with sufficient statistical power to calculate the health risk of COPD from the use of solid fuel, which followed standardised criteria for the diagnosis of COPD and which dealt with confounding factors. The results were pooled by fuel type and country to produce summary estimates using a random effects model. Publication bias was also estimated.

Results There were positive associations between the use of solid fuels and COPD $(\mathrm{OR}=2.80,95 \% \mathrm{Cl} 1.85$ to 4.0) and chronic bronchitis $(\mathrm{OR}=2.32,95 \% \mathrm{Cl} 1.92$ to 2.80). Pooled estimates for different types of fuel show that exposure to wood smoke while performing domestic work presents a greater risk of development of COPD and chronic bronchitis than other fuels.

Conclusion Despite heterogeneity across the selected studies, exposure to solid fuel smoke is consistently associated with COPD and chronic bronchitis. Efforts should be made to reduce exposure to solid fuel by using either cleaner fuel or relatively cleaner technology while performing domestic work.

More than 3 billion people, around half the world's population, use solid fuel to meet their basic household energy demands, and a large proportion of this exposed population lives in less economically developed countries (LEDCs). ${ }^{1}$ In many areas of Africa, Central America, South-east Asia and South Asia $>90 \%$ of rural homes use solid fuel as the primary cooking and/or heating fuel. Women in the rural areas of LEDCs undertake routine cooking and heating over long periods, often many hours per day, and tend to be exposed to much higher levels of indoor air pollutants than men living in the same household. ${ }^{2}$

Chronic obstructive pulmonary disease (COPD), regarded as a disease of developed countries, is now recognised as a common disease in LEDCs. COPD is the fourth leading cause of all deaths, leading to the deaths of $\sim 3$ million people in 2004, of which $90 \%$ of all COPD deaths were from low and middle income countries. ${ }^{3}$ The main contributory factors are cigarette smoke, occupational exposures and exposure to solid fuel smoke. Lower socioeconomic status increases the risk of developing COPD, although which component factors (eg, poor housing, poor nutrition, low income, no/poor education) are the most important in influencing COPD and to what extent is unclear. The use of solid fuel has been suggested as a key causal factor. ${ }^{4-6}$ Although the variation in findings across studies is wide, this could be a real effect due to differing causal influences or could be due to methodological inadequacies. Many studies are observational, small in size with insufficient statistical power to show a clear relationship, with relevant confounding factors often inadequately addressed. A further issue is the use of non-standard definitions of COPD or chronic bronchitis.

In order to describe quantitatively the relationship between COPD and the domestic use of solid fuels, we conducted a systematic literature review and meta-analysis of studies where the methodology fulfilled specific criteria in order to produce an estimate of the capacity of biomass smoke exposure to cause COPD.

\section{METHODS \\ Search strategy}

Using Medline (1980-2009), the Cochrane database, Google scholar (Advanced Google search) and the WHO Library \& Information Networks for Knowledge Database (WHOLIS) we conducted a literature search for COPD, chronic bronchitis and biomass or solid fuel use, using "OR" and "AND" as combining terms for exposure and outcome. The search terms used are summarised in Supplementary table S1. The number of hits and steps in searching the literature from MEDLINE are given in Supplementary table S1 and combined in Supplementary table S2. The same search terms were used for other search engines and databases. References in each of the identified papers were screened for any articles that were not identified in the original search. The aim was to analyse studies which considered COPD defined by spirometry separately from studies which considered chronic bronchitis on the basis of questionnaire responses.

\section{Selection of studies}

The inclusion criteria for the papers to be included in the meta-analysis are given in box 1. A flow diagram for details of the review process, and studies included and excluded is given in figure 1 . The initial approach to inclusion of studies was deliberately wide. We did not use a standard quality tool, but developed a list of critical information which was 
required to be present in each study. Predefined forms were used to extract information from 63 papers, and 23 of them (10 for COPD (table 1), 11 for chronic bronchitis and 2 for both, (table 2)) fulfilled the inclusion criteria and were used in the meta-analysis. Studies without a proper comparator group were discarded.

Selection was undertaken at each stage by two of the authors (OK and JA) and disagreements settled by consensus.
Studies that had defined COPD according to American Thoracic Society (ATS) ${ }^{7}$ and/or Global Initiative for Obstructive Lung Disease (GOLD) ${ }^{8}$ criteria, used the spirometric criterion of a forced expiratory volume in $1 \mathrm{~s}\left(\mathrm{FEV}_{1}\right) /$ forced vital capacity (FVC) ratio $<70 \%$ or on the basis of a diagnosis by a doctor, and studies that had defined chronic bronchitis according to Medical Research Council (MRC) criteria were included in the final

Table 1 Effect size of COPD associated with solid fuel smoke exposure

\begin{tabular}{|c|c|c|c|c|c|c|c|c|c|}
\hline \multirow[b]{2}{*}{ Reference } & \multirow[b]{2}{*}{ Country } & \multirow{2}{*}{$\begin{array}{l}\text { Types of } \\
\text { study }\end{array}$} & \multirow[b]{2}{*}{ Fuel type } & \multirow[b]{2}{*}{ Sample size } & \multirow{2}{*}{$\begin{array}{l}\text { Exposure } \\
\text { measured }\end{array}$} & \multirow[b]{2}{*}{ Types of diagnosis } & \multirow[b]{2}{*}{ Sample type } & \multicolumn{2}{|l|}{ Effect size } \\
\hline & & & & & & & & $\%$ & OR (95\% CI) \\
\hline $\begin{array}{l}\text { Dossing et al } \\
1994^{20}\end{array}$ & $\begin{array}{l}\text { Saudi } \\
\text { Arabia }\end{array}$ & $\begin{array}{l}\text { CC study } \\
\text { defined by } \\
\text { diseases }\end{array}$ & Wood & $\begin{array}{l}50 \text { cases, } 71 \\
\text { controls }\end{array}$ & $\begin{array}{l}\text { No direct } \\
\text { measurement }\end{array}$ & $\begin{array}{l}\mathrm{FEV}_{1} / \mathrm{FVC}<70 \% \text { and } \mathrm{FEV}_{1}<70 \% \text { of } \\
\text { predicted value; }<15 \% \text { or } \\
<250 \mathrm{ml} \text { absolute reduction of } \\
\mathrm{FEV}_{1} \text { after inhalation of } 200 \mu \mathrm{g} \\
\text { aerosolised salbutamol }\end{array}$ & $\begin{array}{l}\text { Adults with } \\
\text { COPD }\end{array}$ & 88 vs 34 & $\begin{array}{l}17.42(10.58 \text { to } \\
28.68)\end{array}$ \\
\hline $\begin{array}{l}\text { Dennis et al } \\
1996^{31}\end{array}$ & Columbia & $\begin{array}{l}\text { CC study } \\
\text { defined by } \\
\text { diseases }\end{array}$ & $\begin{array}{l}\text { Wood and } \\
\text { gasoline }\end{array}$ & $\begin{array}{l}104 \text { cases, } \\
104 \text { controls }\end{array}$ & $\begin{array}{l}\text { No direct } \\
\text { measurement }\end{array}$ & $\begin{array}{l}\mathrm{FEV}_{1} / \mathrm{FVC}<70 \% \text { and } \mathrm{FEV}_{1}<70 \% \\
\text { of predicted value }\end{array}$ & $\begin{array}{l}\text { Women } \\
\geq 35 \text { years } \\
\text { with COPD }\end{array}$ & 85 vs 62 & $3.92(1.7$ to 9.1$)$ \\
\hline $\begin{array}{l}\text { Orozco-Levi } \\
\text { et al } 2006^{16}\end{array}$ & Spain & $\begin{array}{l}\text { CC study } \\
\text { defined by } \\
\text { diseases }\end{array}$ & $\begin{array}{l}\text { Wood }(\mathrm{W}) \text {, } \\
\text { charcoal }(\mathrm{C}) \\
\text { or both }(\mathrm{B})\end{array}$ & $\begin{array}{l}60 \text { cases } \\
\text { (hospitalised), } \\
60 \text { control }\end{array}$ & $\begin{array}{l}\text { Duration of } \\
\text { exposure } \\
\text { reported. }\end{array}$ & $\begin{array}{l}\mathrm{FEV}_{1} / \mathrm{FVC}<70 \% \text { and } \mathrm{FEV}_{1}<80 \% \text { of } \\
\text { predicted value; }<12 \% \text { change in } \\
\mathrm{FEV}_{1} \text { after inhalation of } 400 \mu \mathrm{g} \\
\text { inhaled salbutamol }\end{array}$ & $\begin{array}{l}\text { Women } \\
>50 \text { years } \\
\text { with COPD }\end{array}$ & 95 vs 72 & $\begin{array}{l}\text { W: } 1.8(0.6 \text { to } \\
6.0), C: 1.5(0.5 \\
\text { to } 4.6)), B: 4.5 \\
(1.4 \text { to } 14.2)\end{array}$ \\
\hline \multirow[t]{2}{*}{$\begin{array}{l}\text { Regalado } \\
\text { et al } 2006^{32}\end{array}$} & \multirow[t]{2}{*}{ Mexico } & \multirow[t]{2}{*}{ CS study } & \multirow[t]{2}{*}{$\begin{array}{l}\text { Biomass and } \\
\text { gas }\end{array}$} & \multirow[t]{2}{*}{$\begin{array}{l}\text { Biomass } 778, \\
\text { gas } 67\end{array}$} & \multirow[t]{2}{*}{$\begin{array}{l}\mathrm{PM}_{10} \text { measured } \\
\text { for } 1 \mathrm{~h} \text { during } \\
\text { cooking }\end{array}$} & \multirow[t]{2}{*}{$\begin{array}{l}\text { GOLD (G) criteria for diagnosis } \\
\text { of COPD }\end{array}$} & \multirow[t]{2}{*}{$\begin{array}{l}\text { Non-smoking } \\
\text { adult women }\end{array}$} & $\begin{array}{l}\text { Biomass } \\
\text { G I: } 13.5 \\
\text { G II: } 2.7\end{array}$ & $\begin{array}{l}\text { Biomass: G I } \\
1.5(0.5 \text { to } 4.3)\end{array}$ \\
\hline & & & & & & & & Gas G I: 9.1 & \\
\hline \multirow[t]{5}{*}{$\begin{array}{l}\text { Liu et al } \\
2007^{14}\end{array}$} & \multirow[t]{5}{*}{ China } & \multirow[t]{5}{*}{ CS study } & \multirow{5}{*}{$\begin{array}{l}\text { Biomass } \\
\text { (BM), coal (C) } \\
\text { and LPG }\end{array}$} & $\begin{array}{l}\text { Combined BM } \\
1306\end{array}$ & \multirow{5}{*}{$\begin{array}{l}\text { Measured } \mathrm{SO}_{2} \\
\mathrm{NO}_{2}, \mathrm{CO} \text { and } \\
\mathrm{PM}_{10} \text { directly }\end{array}$} & \multirow[t]{5}{*}{$\begin{array}{l}\text { GOLD criteria for diagnosis } \\
\text { of COPD }\end{array}$} & \multirow[t]{3}{*}{$\begin{array}{l}\text { Adults } \geq \\
40 \text { years }\end{array}$} & 12.0 (BM) & $\begin{array}{l}\text { BM: } 1.72(1.27 \\
\text { to } 2.35)\end{array}$ \\
\hline & & & & C 172 & & & & $12.2(\mathrm{C})$ & \multirow{2}{*}{$\begin{array}{l}\mathrm{C}: 1.55 \\
(0.74 \text { to } 3.22) \\
\mathrm{LPG}=\text { reference }\end{array}$} \\
\hline & & & & \multirow[t]{2}{*}{ LPG 1808} & & & & 7.3 (LPG) & \\
\hline & & & & & & & \multirow{2}{*}{$\begin{array}{l}\text { Non-smoking } \\
\text { women } \\
\geq 40 \text { years }\end{array}$} & 7.3 (BM) & $\begin{array}{l}\text { BM: } 3.11 \\
(1.63 \text { to } 5.94)\end{array}$ \\
\hline & & & & $\begin{array}{l}\text { Non-smoking } \\
\text { women } \\
\text { BM } 744 \\
\text { C } 90 \\
\text { LPG } 995\end{array}$ & & & & $\begin{array}{l}6.7(\mathrm{C}) \\
2.5(\mathrm{LPG})\end{array}$ & $\begin{array}{l}\text { C: } 2.77 \text { ( } 0.83 \text { to } \\
9.26) \text {, } \\
\mathrm{LPG}=\text { Reference }\end{array}$ \\
\hline $\begin{array}{l}\text { Zhong et al } \\
2007^{22}\end{array}$ & China & CS study & Biomass & $\begin{array}{l}10661 \text { (never } \\
\text { exposed); } 9584 \\
\text { (ever exposed) }\end{array}$ & $\begin{array}{l}\text { Not measured } \\
\text { directly }\end{array}$ & $\begin{array}{l}\text { GOLD criteria for diagnosis of } \\
\text { COPD }\end{array}$ & $\begin{array}{l}\text { Adults } \geq \\
40 \text { years }\end{array}$ & $\begin{array}{l}9.3 \text { (ever } \\
\text { exposed), } \\
7.3 \text { (never } \\
\text { exposed) }\end{array}$ & $\begin{array}{l}1.35 \\
(1.20 \text { to } 1.52)\end{array}$ \\
\hline $\begin{array}{l}\text { Caballero } \\
\text { et al } 2008^{33}\end{array}$ & Columbia & CS study & $\begin{array}{l}\text { Wood } \\
\text { exposed } \\
\geq 10 \text { years }\end{array}$ & $\begin{array}{l}2175 \text { (not } \\
\text { exposed); } 3364 \\
\text { (ever exposed) }\end{array}$ & $\begin{array}{l}\text { Not measured } \\
\text { directly }\end{array}$ & $\begin{array}{l}\mathrm{FEV}_{1} / \mathrm{FVC} \text { ratio }<70 \% \text { after } \\
\text { bronchodilator } \\
(200 \mu \mathrm{g} \text { salbutamol) }\end{array}$ & $\begin{array}{l}\text { Adults } \geq \\
40 \text { years }\end{array}$ & $\begin{array}{l}13.4 \% \\
\text { (exposed for } \\
\geq 10 \text { years) }\end{array}$ & $\begin{array}{l}2.42 \text { ( } 2.0 \text { to } \\
2.92) \text { (exposed } \\
\text { for } \geq 10 \text { years) }\end{array}$ \\
\hline $\begin{array}{l}\text { Xu et al } \\
2007^{15}\end{array}$ & China & $\begin{array}{l}\text { CC study } \\
\text { defined by } \\
\text { exposure to } \\
\text { solid fuel } \\
\text { and smoking }\end{array}$ & $\begin{array}{l}\text { Electricity/ } \\
\text { gas, coal, } \\
\text { firewood/ } \\
\text { straw }\end{array}$ & $\begin{array}{l}1743 \text { cases, } \\
1743 \text { controls }\end{array}$ & $\begin{array}{l}\text { No direct } \\
\text { measurement }\end{array}$ & Hospital diagnosed & $\begin{array}{l}\text { Adults } \geq 35 \\
\text { years }\end{array}$ & $\begin{array}{l}74.1 \\
\text { (electricity/ } \\
\text { gas), } 2.7 \\
\text { (coal) and } \\
23.2 \\
\text { (firewood/ } \\
\text { straw) }\end{array}$ & $\begin{array}{l}1.32 \text { (0.79 to } \\
2.20) \text { (coal user) } \\
\text { and } 0.71 \text { ( } 0.46 \\
\text { to } 1.10) \\
\text { (firewood/straw) }\end{array}$ \\
\hline $\begin{array}{l}\text { Chapman } \\
\text { et al } 2005^{34}\end{array}$ & China & $\begin{array}{l}\text { Retrospective } \\
\text { cohort study } \\
\text { (switched to } \\
\text { chimney } \\
\text { stove) }\end{array}$ & Coal & 20453 & $\begin{array}{l}\text { No direct } \\
\text { measurement }\end{array}$ & Hospital diagnosed & $\begin{array}{l}16606(81.2 \%) \\
\text { had chimney } \\
\text { stove and } 3847 \\
(18.8 \%) \text { no } \\
\text { chimney stove }\end{array}$ & $\begin{array}{l}19.4 \% \text { (no } \\
\text { chimney } \\
\text { stove) and } \\
4.5 \% \text { (changed } \\
\text { to chimney } \\
\text { stove) }\end{array}$ & $\begin{array}{l}5.15 \text { (4.62 to } \\
5.74)\end{array}$ \\
\hline $\begin{array}{l}\text { Sezer et al } \\
2004^{35}\end{array}$ & Turkey & $\begin{array}{l}\text { CC study } \\
\text { defined by } \\
\text { diagnosis } \\
\text { of COPD }\end{array}$ & Biomass & $\begin{array}{l}74 \text { COPD cases } \\
\text { and } 74 \text { non- } \\
\text { COPD cases } \\
\text { (healthy) }\end{array}$ & $\begin{array}{l}\text { No direct } \\
\text { measurement }\end{array}$ & Hospital diagnosed & $\begin{array}{l}\text { Non-smoking } \\
\text { women } \\
\geq 38 \text { years }\end{array}$ & $\begin{array}{l}75.7 \% \text { cases } \\
\text { and } 70.3 \% \\
\text { controls } \\
\text { exposed to } \\
\text { biomass }\end{array}$ & $\begin{array}{l}6.61(2.17 \text { to } \\
20.18) \text { in women } \\
\text { exposed to } \\
\text { biomass } \geq 30 \\
\text { years }\end{array}$ \\
\hline $\begin{array}{l}\text { Ekici et al } \\
2005^{36}\end{array}$ & Turkey & $\begin{array}{l}\text { CC study } \\
\text { defined by } \\
\text { fuel types }\end{array}$ & $\begin{array}{l}\text { Biomass and } \\
\text { LPG }\end{array}$ & $\begin{array}{l}\text { Biomass } \\
\text { (cases) }=397 \\
\text { LPG } \\
\text { (control) }=199\end{array}$ & $\begin{array}{l}\text { No direct } \\
\text { measurement }\end{array}$ & $\begin{array}{l}\text { BMRC criteria for the diagnosis } \\
\text { of } \mathrm{CB} \text { and } \mathrm{CAO} \text { defined by } \\
\mathrm{FEV}_{1} / \mathrm{FVC}<70\end{array}$ & $\begin{array}{l}\text { Non-smoking } \\
\text { women }> \\
40 \text { years }\end{array}$ & 23.1 & 2.5 (1.4 to 4.0$)$ \\
\hline $\begin{array}{l}\text { Kiraz et al } \\
2003^{21}\end{array}$ & Turkey & $\begin{array}{l}\text { CC study } \\
\text { defined by } \\
\text { fuel types }\end{array}$ & $\begin{array}{l}\text { Biomass and } \\
\text { LPG }\end{array}$ & $\begin{array}{l}\text { Biomass }=242 \\
\text { and non- } \\
\text { biomass }=102\end{array}$ & $\begin{array}{l}\text { No direct } \\
\text { measurement }\end{array}$ & ATS and BMRC & Adult women & 12.4 vs 3.9 & $\begin{array}{l}28.9(8.7 \text { to } \\
95.9)\end{array}$ \\
\hline
\end{tabular}

ATS, American Thoracic Society; BMRC, British Medical Research Council; CA0, chronic airway obstruction; CB, chronic bronchitis; CC, case-control; COPD, chronic obstructive pulmonary disease; CS, cross-sectional; DS, descriptive study; FEV 1 , forced expiratory volume in 1 s; FVC, forced vital capacity; GOLD, Global Initiative for Obstructive Lung Disease; LPG, liquefied petroleum gas; $\mathrm{PM}_{10}$, particles of $\leq 10 \mu \mathrm{m}$. 
analysis. ${ }^{9}$ Studies from the systematic review which provided neither an effect size nor sufficient information to calculate an effect size were excluded from the meta-analysis.

\section{Statistical methods}

Data were entered into an Excel sheet and then ORs and CI data were extracted from the paper. In the case of papers not reporting ORs but providing sufficient information, ORs were calculated. The ORs and CI data were entered as two separate data sets for COPD and chronic bronchitis in STATA (version 10, STATA, College Station, Texas, USA). The natural log of the ORs and the standard error (SE) of the log OR were used to estimate the pooled effect size of all the studies. Subgroup pooled estimates were calculated based on diagnostic criteria (lung function defined and doctor diagnosed) for COPD, and on country and fuel types for both COPD and chronic bronchitis. ORs were used as they are relatively insensitive to differences in baseline events. ${ }^{10}$ Random effects models were used as effect sizes might be higher (or lower) across studies because of differing age groups, exposure to different fuel smoke and different confounding factors. Within-group heterogeneity was evaluated using $\mathrm{Q}$-tests and $\mathrm{I}^{2}$ statistics. Heterogeneity between different studies was visually explored using Galbraith plots, ${ }^{11}$ and sources of heterogeneity were systematically examined by meta-regression. ${ }^{11}$ Begg's funnel plot ${ }^{12}$ and Egger's test ${ }^{13}$ were used to assess publication bias. Meta-analysis was performed in STATA.

\section{RESULTS}

The initial search revealed 4164 titles of which 1270 abstracts were reviewed, and 184 papers were selected from the abstracts. Twenty-three studies were selected for inclusion in the metaanalysis, the remainder being excluded either because of failure to meet the inclusion criteria or because data were unusable, or both.

Table 2 Effect size of chronic bronchitis associated with solid fuel smoke exposure

\begin{tabular}{|c|c|c|c|c|c|c|c|c|c|}
\hline \multirow[b]{2}{*}{ Reference } & \multirow[b]{2}{*}{ Type of study } & \multirow[b]{2}{*}{ Country } & \multirow[b]{2}{*}{ Fuel type } & \multirow{2}{*}{\multicolumn{2}{|c|}{ Exposure measuredSample size }} & \multirow{2}{*}{$\begin{array}{l}\text { Diagnosis } \\
\text { criteria }\end{array}$} & \multirow[b]{2}{*}{ Sample type } & \multicolumn{2}{|l|}{ Effect size } \\
\hline & & & & & & & & $\%$ & $\mathbf{O R}$ \\
\hline \multirow{3}{*}{$\begin{array}{l}\text { Pandey }(1984 a \\
\text { and } b)^{37,38}\end{array}$} & \multirow[t]{3}{*}{ CS study } & \multirow[t]{3}{*}{ Nepal } & \multirow[t]{3}{*}{ Biomass } & \multirow{3}{*}{$\begin{array}{l}\text { Exposure not } \\
\text { measured directly }\end{array}$} & 748 & BMRC & Non-smoking & $13.76 \%$ (women) & 7.87 (4.67 to \\
\hline & & & & & & criteria & adults $\geq 20$ years & $3.0 \%$ (men) & $13.26)$ \\
\hline & & & & & 2826 & $\begin{array}{l}\text { BMRC } \\
\text { criteria }\end{array}$ & $\begin{array}{l}\text { Adults } \\
\geq 20 \text { years }\end{array}$ & $18.30 \%$ & $\begin{array}{l}\text { Could not be } \\
\text { calculated }\end{array}$ \\
\hline $\begin{array}{l}\text { Albalak et al } \\
(1999)^{39}\end{array}$ & CS study & Bolivia & Biomass & $\begin{array}{l}\mathrm{PM}_{10} \text { exposure } \\
\text { measured directly } \\
\text { in both indoor and } \\
\text { outdoor kitchen }\end{array}$ & $\begin{array}{l}\text { Indoor }=102 \\
\text { Outdoor }=139\end{array}$ & $\begin{array}{l}\text { BMRC } \\
\text { criteria }\end{array}$ & $\begin{array}{l}\text { Adults } \\
\geq 20 \text { years }\end{array}$ & $\begin{array}{l}22 \% \text { (indoor), } \\
13 \% \text { (outdoor) }\end{array}$ & $2.5(1.25$ to 5.00$)$ \\
\hline $\begin{array}{l}\text { Behera and } \\
\text { Jindal }(1991)^{40}\end{array}$ & DS & India & $\begin{array}{l}\text { Biomass, LPG, } \\
\text { kerosene, mixed }\end{array}$ & $\begin{array}{l}\text { Exposure not } \\
\text { measured directly }\end{array}$ & 3608 & $\begin{array}{l}\text { BMRC } \\
\text { criteria }\end{array}$ & $\begin{array}{l}\text { Women } \\
\text { involved } \\
\text { in cooking }\end{array}$ & $\begin{array}{l}2.9 \% \text { (biomass) } \\
1.3 \text { (kerosene), } \\
2.5 \text { (LPG) and } \\
1.2 \text { (mixed) }\end{array}$ & $\begin{array}{l}1.18 \text { (0.83 to } 1.67 \text { ) } \\
\text { (biomass user) }\end{array}$ \\
\hline $\begin{array}{l}\text { Qureshi } \\
(1994)^{41}\end{array}$ & $\begin{array}{l}\text { CC study defined } \\
\text { by fuel(s) used }\end{array}$ & India & $\begin{array}{l}\text { Biomass (BM), } \\
\text { mixed fuel (MF) }\end{array}$ & $\begin{array}{l}\text { Exposure not } \\
\text { measured directly }\end{array}$ & $\begin{array}{l}286 \text { (BM), } \\
560 \text { (MF) }\end{array}$ & $\begin{array}{l}\text { BMRC } \\
\text { criteria }\end{array}$ & $\begin{array}{l}\text { Adults } \\
\geq 15 \text { years }\end{array}$ & $\begin{array}{l}10.14 \%(\mathrm{BM}) \\
5.11 \%(\mathrm{MF})\end{array}$ & $\begin{array}{l}2.10 \text { (1.50 to } 2.94) \\
\text { (biomass user) }\end{array}$ \\
\hline \multirow[t]{4}{*}{$\begin{array}{l}\text { Akhtar et al } \\
(2007)^{18}\end{array}$} & \multirow[t]{4}{*}{$\begin{array}{l}\text { CC study defined } \\
\text { by fuel types }\end{array}$} & \multirow[t]{4}{*}{ Pakistan } & $\begin{array}{l}\text { Biomass mixed } \\
(\mathrm{BM}) \text {, }\end{array}$ & \multirow[t]{4}{*}{$\begin{array}{l}\text { Exposure not } \\
\text { measured directly }\end{array}$} & \multirow[t]{4}{*}{$\begin{array}{l}1426 \text { cases and } \\
1131 \text { controls }\end{array}$} & \multirow[t]{4}{*}{$\begin{array}{l}\text { ATS } \\
\text { criteria }\end{array}$} & \multirow[t]{4}{*}{$\begin{array}{l}\text { Female non- } \\
\text { smokers }\end{array}$} & \multirow{4}{*}{$\begin{array}{l}7.01 \% \text { (cases) } \\
\text { and } 2.92 \% \\
\text { (control) }\end{array}$} & $\begin{array}{l}2.51 \text { (1.65 to } 3.83) \\
\text { (BM) }\end{array}$ \\
\hline & & & Wood (W), & & & & & & $\begin{array}{l}2.38(2.12 \text { to } 3.01) \\
\text { (W) }\end{array}$ \\
\hline & & & Dung (D) & & & & & & $\begin{array}{l}2.01 \text { (1.72 to } 2.42) \\
\text { (D) }\end{array}$ \\
\hline & & & Straw (S) & & & & & & $\begin{array}{l}3.32 \text { (1.11 to } 9.88) \\
\text { (S) }\end{array}$ \\
\hline \multirow{3}{*}{$\begin{array}{l}\text { Menezes } \\
(1994)^{17}\end{array}$} & \multirow[t]{3}{*}{ CS survey } & \multirow[t]{3}{*}{ Brazil } & \multirow{3}{*}{$\begin{array}{l}\text { Solid fuel and } \\
\text { clean fuel used }\end{array}$} & \multirow{3}{*}{$\begin{array}{l}\text { Exposure not } \\
\text { measured directly } \\
\text { but classified as } \\
\text { high, moderate } \\
\text { and no exposure }\end{array}$} & 411 (none) & \multirow{3}{*}{$\begin{array}{l}\text { ATS } \\
\text { criteria }\end{array}$} & \multirow{3}{*}{$\begin{array}{l}\text { Adults } \\
\geq 40 \text { years }\end{array}$} & $9.3 \%$ (mone) & 1.0 (none) \\
\hline & & & & & 385 (moderate) & & & $14.0 \%$ (moderate) & $1.49(0.92$ to 2.41$)$ \\
\hline & & & & & 257 (high) & & & $16.0 \%$ (high) & $1.30(0.75$ to 2.27$)$ \\
\hline $\begin{array}{l}\text { Ekici et al } \\
(2005)^{36}\end{array}$ & $\begin{array}{l}\text { CC study defined } \\
\text { by fuel types }\end{array}$ & Turkey & $\begin{array}{l}\text { Biomass and } \\
\text { LPG }\end{array}$ & $\begin{array}{l}\text { Exposure not } \\
\text { measured directly }\end{array}$ & $\begin{array}{l}397 \text { (cases) } 199 \\
\text { (control) }\end{array}$ & $\begin{array}{l}\text { BMRC } \\
\text { criteria }\end{array}$ & $\begin{array}{l}\text { Non-smoking } \\
\text { women } \\
\geq 40 \text { years }\end{array}$ & $\begin{array}{l}28.5 \% \text { (cases) } \\
13.6 \% \text { (controls) }\end{array}$ & $2.5(1.5$ to 4.0$)$ \\
\hline $\begin{array}{l}\text { Kiraz et al } \\
(2003)^{21}\end{array}$ & $\begin{array}{l}\text { CC study defined } \\
\text { by fuel types }\end{array}$ & Turkey & $\begin{array}{l}\text { Biomass and } \\
\text { LPG }\end{array}$ & $\begin{array}{l}\text { Exposure not } \\
\text { measured directly }\end{array}$ & $\begin{array}{l}242 \text { (cases) } 102 \\
\text { (control) }\end{array}$ & $\begin{array}{l}\text { BMRC } \\
\text { criteria }\end{array}$ & $\begin{array}{l}\text { Women } \\
\geq 25 \text { years }\end{array}$ & $\begin{array}{l}20.7 \% \text { (cases) } \\
10.8 \% \text { (control) }\end{array}$ & $1.9(1.3$ to 3.01$)$ \\
\hline $\begin{array}{l}\text { Uzun et al } \\
2003^{42}\end{array}$ & CS study & Turkey & $\begin{array}{l}\text { Biomass and } \\
\text { non-biomass }\end{array}$ & $\begin{array}{l}\text { No direct } \\
\text { measurement }\end{array}$ & $\begin{array}{l}90 \text { (biomass) } \\
87 \text { (non-biomass) }\end{array}$ & $\begin{array}{l}\text { BMRC } \\
\text { criteria }\end{array}$ & Adult women & $\begin{array}{l}58.9 \% \text { (biomass) } \\
29.4 \% \text { (non- } \\
\text { biomass) }\end{array}$ & $3.36(1.80$ to 6.26$)$ \\
\hline $\begin{array}{l}\text { Cetinkaya } \\
\text { et al } 2000^{43}\end{array}$ & CS study & Turkey & Biomass & $\begin{array}{l}\text { No direct } \\
\text { measurement }\end{array}$ & $\begin{array}{l}461 \text { (men) } \\
562 \text { (women) }\end{array}$ & $\begin{array}{l}\text { ERS } \\
\text { criteria }\end{array}$ & $\begin{array}{l}\text { Adult men vs } \\
\text { women }\end{array}$ & $17.8 \%$ vs $10.0 \%$ & 1.96 (1.36 to 2.82$)$ \\
\hline $\begin{array}{l}\text { Golshan et al } \\
2002 b^{44}\end{array}$ & CS study & Iran & $\begin{array}{l}\text { Gas, kerosene } \\
\text { and wood }\end{array}$ & $\mathrm{PM}_{10}$ measured & $\begin{array}{l}470 \text { (gas) } \\
493 \text { (kerosene) } \\
227 \text { (wood) }\end{array}$ & $\begin{array}{l}\text { BMRC } \\
\text { criteria }\end{array}$ & Women & $3.4 \%$ & $\begin{array}{l}2.91(2.08 \text { to } 4.4) \\
\text { for using wood in } \\
\text { the past }\end{array}$ \\
\hline $\begin{array}{l}\text { Dutt et al } \\
1996^{45}\end{array}$ & CS study & India & $\begin{array}{l}\mathrm{LPG} \text {, kerosene, } \\
\text { biomass }\end{array}$ & $\begin{array}{l}\text { No direct } \\
\text { measurement }\end{array}$ & $\begin{array}{l}97 \text { (biomass) } \\
100 \text { (kerosene) } \\
98 \text { (LPG) }\end{array}$ & $\begin{array}{l}\text { BMRC } \\
\text { criteria }\end{array}$ & $\begin{array}{l}\text { Women } \\
15-60 \text { years }\end{array}$ & $\begin{array}{l}4.1 \% \text { (biomass) } \\
2.0 \% \text { (kerosene) } \\
1.0 \% \text { (LPG) }\end{array}$ & $\begin{array}{l}4.17(0.46 \text { to } \\
38.02) \\
\text { (biomass vs LPG) }\end{array}$ \\
\hline $\begin{array}{l}\text { Perez-Padilla } \\
\text { et al } 1996^{46}\end{array}$ & $\begin{array}{l}\text { CS study (defined } \\
\text { by disease type) }\end{array}$ & Mexico & Wood & $\begin{array}{l}\text { No direct } \\
\text { measurement }\end{array}$ & $\begin{array}{l}127 \text { (CB or CAO) } \\
375 \text { controls with } \\
\text { other diseases }\end{array}$ & $\begin{array}{l}\text { Hospital } \\
\text { diagnosed }\end{array}$ & $\begin{array}{l}\text { Women } \\
>40 \text { years }\end{array}$ & $\begin{array}{l}76 \% \text { of individuals } \\
\text { with CB were } \\
\text { exposed to wood } \\
\text { smoke }\end{array}$ & $3.9(2.0$ to 7.6$)$ \\
\hline
\end{tabular}

ATS, American Thoracic Society; BMRC, British Medical Research Council; CA0, chronic airway obstruction; CB, chronic bronchitis; CC, case-control; CS, cross-sectional; DS, descriptive study; ERS, European Respiratory Society; $L P G$, liquefied petroleum gas; $\mathrm{PM}_{10}$, particles of $\leq 10 \mu \mathrm{m}$. 
Summary tables of studies included are shown for COPD in table 1 and for chronic bronchitis in table 2. Most were case-control studies defined by exposure or fuel used, although few studies provided direct exposure assessment, exposure being defined by proxy methods such as the number of hours spent cooking. Five hospital-based case-control studies selected controls from patients visiting the outpatient department with ill-defined illness for whom exposures were unclear. Three studies $^{14-16}$ in the COPD group of papers and two studies ${ }^{17} 18$ in the chronic bronchitis group reported effect sizes for different types of solid fuel. For these studies (figure 2 and figure 3), results for each individual type of biomass are recorded separately.

\section{Effect estimates}

COPD

Random effect models were used to calculate the pooled effect size for both lung function-defined (Q-statistic $=157.62$ (degrees of freedom $(\mathrm{df}=13)), \mathrm{p}<0.001 ; \mathrm{I}^{2}=91.8 \%$; tau-squared $=0.3936$ and $\mathrm{Z}=5.49, \mathrm{p}<0.001)$ ) and doctor-diagnosed ( $\mathrm{Q}$-statistic $=96.04$ $(\mathrm{df}=3), \mathrm{p}<0.001 ; \mathrm{I}^{2}=96.9 \%$; tau-squared $=1.3727$ and $\mathrm{Z}=1.36$, $\mathrm{p}=0.173)$ COPD because of heterogeneity across studies. The random pooled effect sizes (OR) for lung function-defined and hospital-diagnosed COPD were 2.96 (95\% CI=2.01 to 4.37) and $2.29(95 \% \mathrm{CI}=0.70$ to 7.52$)$, respectively (figure 2 ).

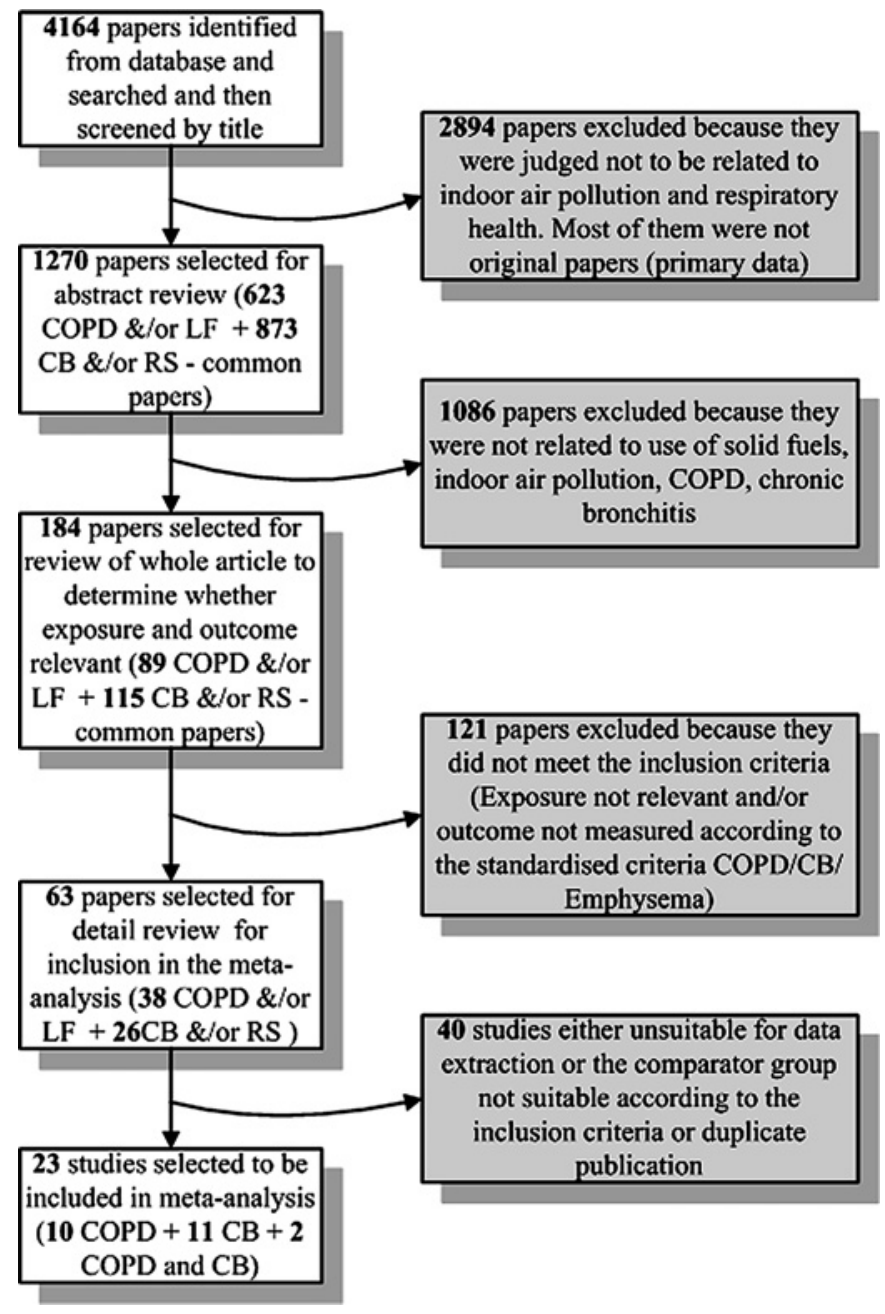

Figure 1 Flow chart showing studies related to chronic obstructive pulmonary disease (COPD) and exposure to solid fuel. CB, chronic bronchitis; LF, lung function; RS, respiratory symptoms.
Subgroup meta-analysis for different fuel types was also conducted using random effect models only for lung functiondefined COPD. The effect size was greater for wood burners $(\mathrm{OR}=4.29,95 \%$ CI 1.35 to 13.70$)$ (figure 4) than for populations using mixed biomass fuels (COPD: $\mathrm{OR}=2.49,95 \%$ CI 1.54 to 4.01). All studies reporting COPD in relation to coal use were from China, with an estimated pooled effect size (OR) of 1.84 (95\% CI 1.01 to 3.35 ) (figure 4). Biomass fuel contributed $46.50 \%$ to the pooled effect size of COPD, followed by wood $(30.10 \%)$ and coal (12.65\%).

\section{Chronic bronchitis}

There was heterogeneity across the studies ( $Q$-statistic $=51.42$ $(\mathrm{df}=16), \mathrm{p}<0.001 ; \mathrm{I}^{2}=68.9 \%$; tau-squared $=0.0942$ and $\mathrm{Z}=8.67$, $\mathrm{p}<0.001)$ and the random pooled effect size was $2.32(95 \% \mathrm{CI}$ 1.92 to 2.80) (figure 3). Wood burners showed a greater effect than mixed biomass fuel burners (wood burners, $\mathrm{OR}=2.64,95 \%$ CI 2.12 to 3.29; mixed biomass fuel burners, $\mathrm{OR}=2.46,95 \% \mathrm{CI}$ 1.78 to 3.39 ) (figure 5).

There is heterogeneity in the pooled risk estimates for both COPD and chronic bronchitis across studies in different countries (Supplementary figures S1 and S4). The result may be due to variation in different types of fuel use (figures 4 and 5) and also the design of the stoves used for cooking activities, as different stoves produce different exposures. ${ }^{19}$

\section{Publication bias}

Publication bias was tested using funnel plots for both COPD (Supplementary figure S2) and chronic bronchitis (Supplementary figure S5). The funnel plot shows that the studies related to most risk estimates were distributed symmetrically along the midline which is the pooled estimate. Five studies (two to the left and three to the right) related to COPD exceeded the $95 \%$ $\mathrm{CI}$, and the same was the case for two studies (one to the left and one to the right) related to chronic bronchitis. The combined data obtained from Egger's test for both COPD (not separated by diagnostic criteria) (bias $=0.99, \mathrm{p}>1 ; \mathrm{t} \mid ;=0.602$ ) and chronic bronchitis (bias=1.14, $\mathrm{p}>|; \mathrm{t}| ;=0.256$ ) showed no evidence of publication bias (Supplementary figures S7 and S8). COPD studies which used the lung function diagnostic criterion showed substantial publication bias (bias=2.5. $\mathrm{p}>|; \mathrm{t}| ;=0.021$ ). Publication bias did not exist for studies diagnosed on lung function criteria when three studies ${ }^{20-22}$ that were outliers in the Galbraith plot were removed (bias $=0.0208, \mathrm{p}>|; \mathrm{t}| ;=0.968$ and $\left.\mathrm{I}^{2}=0.0 \%, \mathrm{p}=0.496\right)$.

\section{Heterogeneity by meta-regression}

Heterogeneity was explored further by meta-regression to assess possible contributions by different factors. Analysis addressed confounding factors (divided into two groups, first adjusting for smoking and age and separately adjusting for more variables (occupational dust exposure, socioeconomic status, income, education) including age and smoking), year of publication (grouped in those before and after 2000), year in which the study was carried out (grouped in those before and after 2000) and sample size. For studies diagnosed on lung function criteria, only year of publication (coefficient $-0.1073, \mathrm{p}>|; \mathrm{t}| ;=0.025$ ) and the year the study was conducted (coefficient $=-1.3107, p>|; t| ;=0.008$ ) were significant contributors to heterogeneity (Supplementary figures S12 and S13). Heterogeneity tests in the same way for studies of chronic bronchitis showed no significant results.

\section{DISCUSSION}

Exposure to solid fuel smoke has been considered as an important risk for COPD and chronic bronchitis in adults of LEDCs. 
Figure 2 Forest plot of studies reporting chronic obstructive pulmonary disease due to exposure to solid fuels (separated by diagnostic criteria).
Studies separated by diagnosis criteria Reference - Country (Fuelused)

OR(95\%Cr)

COPD - Lung Function

Kiraz et al 2003 -Turkey (Biomass)

Dossing et al 1994 - Saudi Arabia (Wood)

Orozco-Levi et al 2006a - Spain (Wood and charcoal)

Dennis et al 1996 - Columbia (Wood)

Liu et al 2007a - China (Biomass)

Liu et al 2007b - China (Coal)

Ekici et al 2005 - Turkey (Biomass)

Caballero et al 2008 - Columbia (Wood)

Orozco-Levi et al 1996b - Spain (Wood)

Liu et al 2007c - China (Biomass)

Liu et al 2007d - China (Coal)

Regaldo et al 2006 - Mexico (Biomass)

Orozco-Levi et al 2006c - Spain (Charcoal)

Zhong et al 2007 - China (Biomass)

Subtotal (I-squared $=91.8 \%, \mathrm{p}<0.001$ )

COPD - Doctor diagnosed

Sezer et al 2004 - Turkey (Biomass)

Chapman et al 2005 - China (Coal)

Xu et al 2007a - China (Coal)

$\mathrm{Xu}$ et al 2007b - China (Firewood/straw)

Subtotal (I-squared $=96.9 \%, p<0.001$ )

Overall (I-squared $=97.3 \%, p<0.001$ )

NOTE: Weights are from rendom encts malysis
$2890(8.71,9590) \quad 434$ $17.40(1055.28 .70) \quad 621$ $450(1.43 .1420) \quad 4.47$ $390(167,9.10) \quad 531$ $3.10(1.63,590) \quad 586$ $280(084.930) \quad 433$ $250(156,400) \quad 627$ $2.40(199,290) \quad 6.73$ $180(054,600) \quad 433$ $1.70(120,2.40)$ $1.60(080.320) \quad 5.73$ $150(052.430) \quad 4.73$ $150(0.49 .460) \quad 455$ $1.40(131,150) \quad 681$ $296(201.437) \quad 76.18$

$6.60(2.16 .2020) \quad 455$ $520(4.24,5.50) \quad 680$ $130(0.77,220) \quad 6.15$ $0.70(0.45,1.10) \quad 631$ $229(0.70 .752) \quad 23.82$ $280(185,423) \quad 100.00$
This meta-analysis has shown that exposure to solid fuel smoke is consistently associated with COPD and chronic bronchitis, regardless of the types of fuel exposure, with overall risks more than double compared with non-biomass fuel users.

Cigarette smoke $e^{23}$ and occupational exposures ${ }^{24}$ have been identified as the main contributory factors to COPD in more developed countries. Populations with lower socioeconomic status have a greater risk of developing COPD, ${ }^{25-27}$ although which component factors (eg, poor housing, poor nutrition, low income, no/poor education) are the most important and to what extent is unclear. Exposure to solid fuel smoke is also considered a major contributory factor, although this belief has been based on surrogate measures of exposure and no studies have shown a relationship between direct measurement of biomass smoke exposure and the prevalence of COPD.

In this meta-analysis, pooled estimates by fuel type showed that wood smoke $(\mathrm{OR}=4.29,95 \%$ CI 1.35 to 13.70$)$ is the most important risk factor for COPD, followed by mixed biomass $(\mathrm{OR}=2.49,95 \% \mathrm{CI} 1.54$ to 4.01$)$ and coal $(\mathrm{OR}=1.84,95 \% \mathrm{CI} 1.01$ to 3.35 ). However, there is clear variation between the different
Figure 3 Forest plot of studies reporting effect size on chronic bronchitis due to exposure to solid fuels.

\begin{tabular}{|c|c|c|c|}
\hline Studies & & & $\%$ \\
\hline Reference -Country & & OR (OS\% CD) & weighe \\
\hline Pandey 1984(Nepal) & $\longrightarrow$ & $78.87(4.67,13.26)$ & 5.70 \\
\hline Dutt et al 1996 (India) & & $4: 17(0.46,38.02)$ & 0.69 \\
\hline Perez-Padilla et al 1996 (Mexico) & & $3: 90(2.00,7.60)$ & 4.48 \\
\hline Uzun et al 2003 (Turkey) & & $3.36(1.80,6.26)$ & 4.83 \\
\hline Akhtar et al 2007a (Pakistan) & & $3: 32(1.12,9.88)$ & 2.33 \\
\hline Golshan et al 2002b (Iran) & & $2.91(1.92,4.40)$ & 6.79 \\
\hline Akhtar et $2007 \mathrm{~b}$ (Pakistan) & & $2.51(1.64,3.83)$ & 6.69 \\
\hline Ekici et al 2005 (Turkey) & & $2.50(1.56,4.00)$ & 6.20 \\
\hline Albalak et al 1999 (Bolivia) & & $2.50(1.25,5.00)$ & 4.29 \\
\hline Akhtar et al 2007c (Pakistan) & & $2.38(1.88,3.01)$ & 8.67 \\
\hline Qureshi 1994 (India) & & $2: 10(1.50,2.94)$ & 7.61 \\
\hline Akhtar et al $2007 \mathrm{~d}$ (Pakistan) & & $2.01(1.67,2.42)$ & 9.12 \\
\hline Cetinkaya et al 2000 (Turkey) & & $1.96(1.36,2.82)$ & 7.32 \\
\hline Kiraz et al 2003 (Turkey) & & $1: 90(1.20,3.01)$ & 6.30 \\
\hline Menezes 1994a(Brazii) & & $1: 49(0.92,2.41)$ & 6.10 \\
\hline Menezes 1994b (Brazii) & & $1: 30(0.74,2.27)$ & 5.38 \\
\hline Behera and Jindal 1991 (India) & & $1: 18(0.83,1.67)$ & 7.49 \\
\hline Overall (I-squared $=68.9 \%, p<0.001$ ) & & $2.32(1.92,2.80)$ & 100.00 \\
\hline NOTE: Weifots are foum rendom efects malysis & & & \\
\hline
\end{tabular}


Figure 4 Forest plot showing the effect size of chronic obstructive pulmonary disease due to exposure to fuel types (for studies based on lung function diagnosis). N/A, not available.
Figure 5 Forest plot showing the effect size on chronic bronchitis due solid fuel exposure. N/A, not available.
Studies (diagnosed by lung function) separated by fuel used Reference - Country

$\mathrm{OR}(95 \% \mathrm{CI})$

$\%$

Biomass

Kiraz et al 2003 -Turkey

Lin et al 2007a - China

Ekici et al 2005 - Turkey

Liv et al 2007c - China

Regaldo et al 2006 - Mexico

Zhong et al 2007 - China

Subtotal (I-squared $=86.2 \%, p<0.001$ )

Wood

Dossing et al 1994 - Saudi Arabia

Dennis et al 1996 - Columbia

Caballero et al 2008 - Columbia

Orozco-Levi et al 1996b - Spain

Subtotal (I-squared $=94.4 \%, p<0.001$ )

Coal

Lin et al 2007b - China

Lin et al 2003d - Ching

Subtotal (I-squared $=0.0 \%, p=0.429$ )

Wood and charcoal

Orozco-Leviet al 2006a - Spain

Subtotal (I-squared $=\mathrm{N} / \mathrm{A} \quad \mathrm{p}=\mathrm{N} / \mathrm{A}$ )

Charcoal

Orozco-Levi et al 2006c - Spain

Subtotal $($ I-squared $=$ N/A $p=N / A)$

Overall (I-squared $=91.8 \%, p<0.001$ )

NOTE: Weights axe from random effects analysis

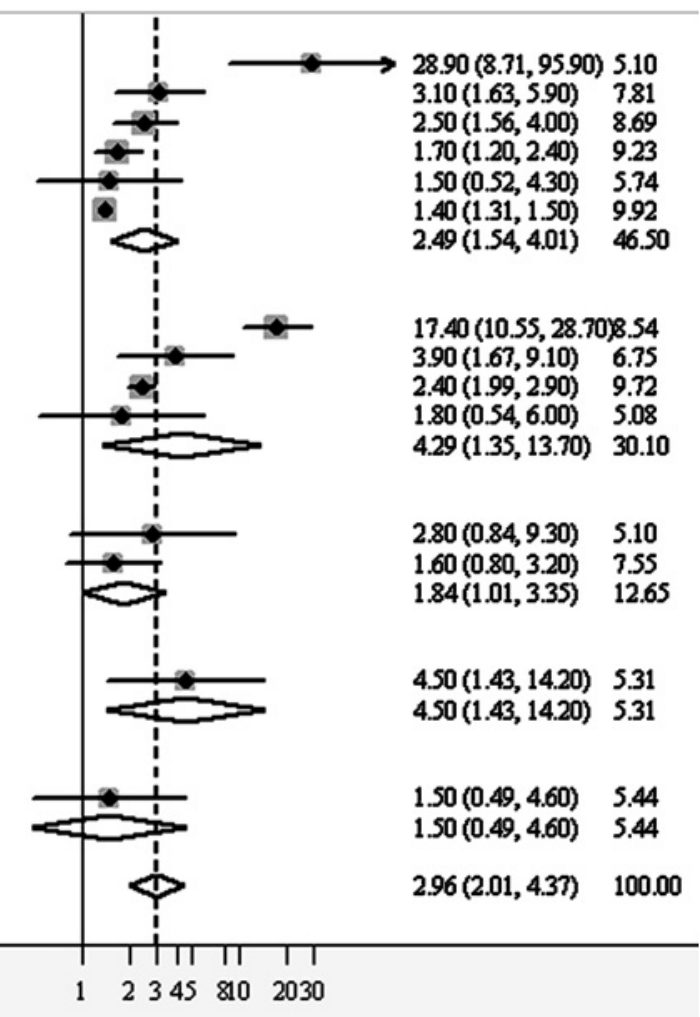

Log of Odds ratio

Studies

Reference - Country

OR $(95 \% \mathrm{CI}) \quad \stackrel{\%}{\text { Weight }}$

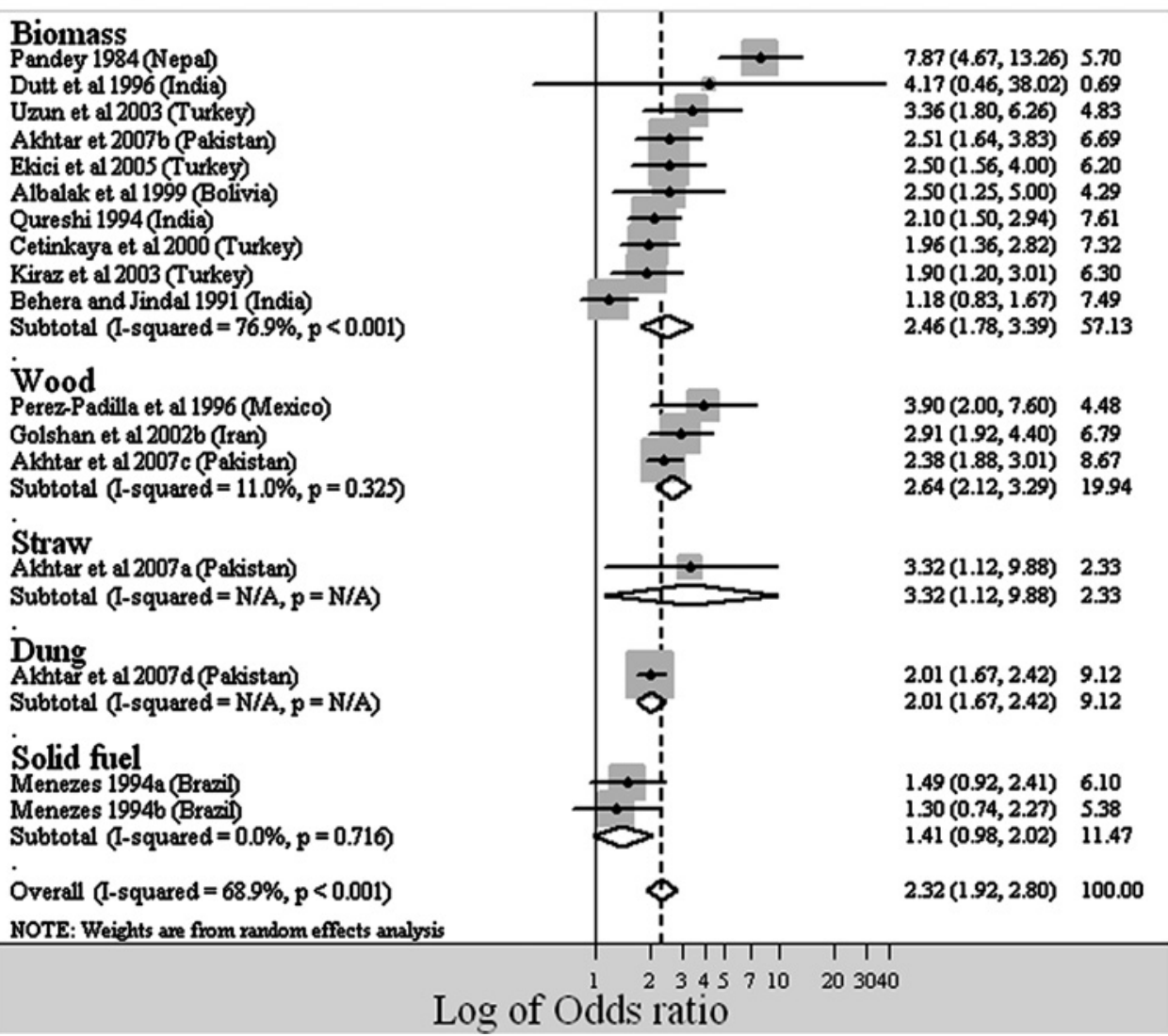


studies included in this meta-analysis in terms of design, dealing with confounders, calculating risk with different comparator groups and exposure assessment. All these factors added substantial statistical heterogeneity between different studies that might have influenced the risk estimate. Almost all the studies of COPD considered populations over the age of 35 years which might increase the effect size as the prevalence of COPD increases with age and is highest among the elderly. ${ }^{28}$ There was variation in the assessment of exposure, most studies not measuring exposure directly, instead using proxy measurements such as the number of hours spent doing cooking or the presence or absence of room ventilation. This lack of direct measurement could have resulted in either overestimation or underestimation of effect, always assuming that current measurement (which, when obtained, is usually measured for a period of $\leq 24 \mathrm{~h}$ ) truly represents long-term exposure which is more relevant in the development of COPD

The pooled estimate of chronic bronchitis for different types of fuel showed similar results to that of COPD, identifying wood smoke $(\mathrm{OR}=2.64,95 \% \mathrm{CI} 2.12$ to 3.29$)$ as the most important risk factor, followed by mixed biomass smoke $(\mathrm{OR}=2.46,95 \% \mathrm{CI} 1.78$ to 3.39$)$. The studies relating to chronic bronchitis used respiratory questionnaires (mostly the British MRC questionnaire): studies which did not use validated questionnaires were excluded from the meta-analysis. Reporting and recall bias cannot be ruled out in questionnaire studies. People in developing countries consider wheeze, breathlessness and bringing up phlegm to some extent as normal, which will probably result in under-reporting of symptoms ${ }^{29}$ and, if this is differentially expressed between exposed and non-exposed groups, this may influence the true risk. Bias might also have occurred in these studies due to use of terminology. Some words such as "wheeze" do not have an equivalent word in many languages. Further, in some parts of the world, people are reluctant to provide personal information, especially regarding health, because of social taboos, especially an issue in southern Asia. Finally, the review was limited by only including studies in English, which might have resulted in publication bias.

In LEDCs, a family switching from one fuel to another is quite common. Many individuals in urban areas who currently use cleaner fuel may, in earlier years, have used solid fuel for cooking. This might have resulted in a residual effect in the control group, so information on past fuel use should be gathered in such studies.

Publication bias was shown in studies of COPD using the lung function criterion showing higher effects in studies which were conducted before 2000. This might relate to study design issues which were not explicit in the described methodology, such as approaches to recruitment, level of training of technicians performing spirometry, differences in use of bronchodilators in assessing COPD or inconsistent use of or lack of calibration of lung function equipment. For both COPD and chronic bronchitis, inadequate allowance for confounders such as exposure to outdoor air pollution or to toxic substances such as pesticides (most rural dwellers are farmers or housewives) or inadequate assessment of cigarette smoke exposure whether active or passive may have resulted in biased results. Finally, the heterogeneity could be real, one explanation being differential toxicity of the smoke from different biomass fuels. ${ }^{30}$

No studies provided information to construct a dose-response function. This should be regarded as an important aim as a simple linear relationship as is seen in studies of outdoor air pollution is unlikely to hold for exposures as high as those recorded in these studies. Determining the shape of the dose-response curve will inform on the levels to which exposures need to be reduced to

\section{Box 1 Inclusion criteria for the systematic review}

1. English language only.

2. Papers containedoriginal data and were full-length peerreviewed reports of cohort, case-control or cross-sectional studies.

3. Data for a non-exposed comparator group were provided.

4. Adjustment for confounding by smoking was addressed.

5. Contained quantitative effect estimates of the associations between exposed and non-exposed group to biomass/solid fuel or data sufficient to calculate effect estimate.

6. Had an adequate sample size.

7. Had used standard questionnaires or well reported questions to measure the respiratory symptoms for chronic bronchitis.

8. Lung function measured according to American Thoracic Society criteria.

have a significant impact on health, and this may vary for different fuels given the heterogeneity by fuel use shown in this analysis.

Future studies need to be designed to include all the important risk factors, especially some form of measurement of direct exposure to biomass smoke. What such a measurement should be is as yet undetermined, but, for the moment, $24 \mathrm{~h}$ mean exposures (as are used in studies of outdoor air pollution exposure) should be regarded as the most appropriate. ${ }^{2}$ Confounders such as environmental tobacco smoke exposure, occupational exposure to dusty environments and smoking status should all be considered. Fuel use history should be recorded accurately to allow life-course exposure to biomass fuel smoke to be modelled or estimated to enable determination of any residual effect of previous solid fuel smoke exposure. Health outcomes should be measured by validated instruments (whether lung function equipment used by well-trained technicians or questionnaires) with clearly defined and agreed protocols adhering to standard criteria.

\section{CONCLUSION}

Despite heterogeneity in design, measurement and quantitative effect estimates among the studies included in this meta-analysis, consistent evidence was found that exposure to indoor air pollution is a risk factor for COPD and chronic bronchitis, with at least a doubling of risk, although with marked heterogeneity by both country and fuel type. At present there is insufficient information to define a dose-response relationship, and there are no epidemiological studies which have considered potential differential toxicity for different fuel types, although this analysis shows a gradient of effect by fuel, with wood smoke being associated with the greatest effect. Future studies should address these study design issues to improve the risk estimates for exposure to different fuel types.

Acknowledgements The authors thank Professor Tom Sorahan for providing statistical advice during the preparation of this manuscript. OK was supported by a PhD studentship awarded by the University of Aberdeen.

Funding University of Birmingham.

Competing interests None.

Provenance and peer review Not commissioned; externally peer reviewed.

\section{REFERENCES}

1. World Health Organization. Fuel for life-household energy and health. [database on the Internet]. World Health Organization. 2006. http://www.who.int/indoorair/ publications/fuelforlife.pdf. 
2. Kurmi OP, Semple S, Steiner M, et al. Particulate matter exposure during domestic work in Nepal. Ann Occup Hyg 2008;52:509-17.

3. World Health Organization. The global burden of disease: update 2004. Geneva: World Health Organization, 2008. http://www.who.int/healthinfo/global_burden_ disease/GBD_report_2004update_full.pdf

4. Bruce $\mathbf{N}$, Perez-Padilla $R$, Albalak $\bar{R}$. The health effects of indoor air pollution exposure in developing countries 2002; WHO/SDE/OEH/02.05: http://whqlibdoc.who.int/hq/ 2002/WHO SDE OEH 02.05.pdf

5. Pandey M $\overline{\mathbf{R}}$. Prevalence of chronic bronchitis in a rural community of the Hill Region of Nepal. Thorax 1984;39:331-6.

6. Smith KR, Samet JM, Romieu I, et al. Indoor air pollution in developing countries and acute lower respiratory infections in children. Thorax 2000;55:518-32.

7. Celli BR, MacNee W, Agusti A, et al. Standards for the diagnosis and treatment of patients with COPD: a summary of the ATS/ERS position paper. Eur Respir $J$ 2004;23:932-46.

8. Rabe KF, Hurd S, Anzueto A, et al. Global strategy for the diagnosis, management, and prevention of chronic obstructive pulmonary disease: gold executive summary. Am J Respir Crit Care Med 2007:176:532-55.

9. Definition and classification of chronic bronchitis for clinical and epidemiological purposes. A report to the Medical Research Council by their Committee on the Aetiology of Chronic Bronchitis. Lancet 1965;1:775-9.

10. Borenstein M, Hedges LV, Higgins JPT, et al. Introduction to meta-analysis. London: John Wiley and Sons, Ltd, 2009.

11. Song F, Sheldon TA, Sutton AJ, et al. Methods for exploring heterogeneity in metaanalysis. Eval Health Prof 2001;24:126-51.

12. Sterne JAC, Egger M, Smith GD. Systematic reviews in health care: investigating and dealing with publication and other biases in meta-analysis. BMJ 2001;323:101-5.

13. Egger M, Smith GD, Schneider M, et al. Bias in meta-analysis detected by a simple, graphical test. BMJ 1997;315:629-34.

14. Liu S, Zhou $Y$, Wang $X$, et al. Biomass fuels are the probable risk factor for chronic obstructive pulmonary disease in rural South China. Thorax 2007:62:889-97.

15. $\mathbf{X u} \mathbf{F}$, Yin $\mathrm{XM}$, Shen $\mathrm{HB}$, et al. Better understanding the influence of cigarette smoking and indoor air pollution on chronic obstructive pulmonary disease: a case-control study in Mainland China. Respirology 2007:12:891-7.
16. Orozco-Levi M, Garcia-Aymerich J, Villar J, et al. Wood smoke exposure and risk of chronic obstructive pulmonary disease. Eur Respir J 2006;27:542-6.

17. Menezes AM, Victora CG, Rigatto M. Prevalence and risk factors for chronic bronchitis in Pelotas, RS, Brazil: a population-based study. Thorax 1994; 49:1217-21.

18. Akhtar T, Ullah Z, Khan MH, et al. Chronic bronchitis in women using solid biomass fuel in rural Peshawar, Pakistan. Chest 2007:132:1472-5.

19. Bruce N, McCracken J, Albalak R, et al. Impact of improved stoves, house construction and child location on levels of indoor air pollution exposure in young Guatemalan children. J Expo Anal Environ Epidemiol 2004;14:S26-33.

20. Dossing M, Khan J, al-Rabiah F. Risk factors for chronic obstructive lung disease in Saudi Arabia. Respir Med 1994:88:519-22.

21. Kiraz K, Kart L, Demir R, et al. Chronic pulmonary disease in rural women exposed to biomass fumes. Clin Invest Med 2003;26:243-8.

22. Zhong N, Wang C, Yao W, et al. Prevalence of chronic obstructive pulmonary disease in China: a large, population-based survey. Am J Respir Crit Care Med 2007:176:753-60.

23. Silverman EK. Genetic epidemiology of COPD. Chest 2002;121:1S-a-6.

24. Balmes J, Becklake $\mathrm{M}$, Blanc $\mathrm{P}$, et al. American thoracic society statement: occupational contribution to the burden of airway disease. Am J Respir Crit Care Med 2003;167:787-97.

25. Prescott E, Lange P, Vestbo J. Socioeconomic status, lung function and admission to hospital for COPD: results from the Copenhagen City Heart Study. Eur Respir J 1999;13:1109-14.

26. Prescott E, Vestbo J. Socioeconomic status and chronic obstructive pulmonary disease. Thorax 1999:54:737-41

27. Tao X, Hong CJ, Yu S, et al. Priority among air pollution factors for preventing chronic obstructive pulmonary disease in Shanghai. Sci Total Environ 1992;127:57-67.

28. Mannino DM, Buist AS. Global burden of COPD: risk factors, prevalence, and future trends. Lancet 2007;370:765-73.

29. Kroeger A. Health interview surveys in developing countries: a review of the methods and results. Int J Epidemiol 1983;12:465-81.

30. Kocbach A, Namork E, Schwarze PE. Pro-inflammatory potential of wood smoke and traffic-derived particles in a monocytic cell line. Toxicology 2008;247:123-32.

\section{Lung alert}

\section{Iron supplementation may attenuate the effects of hypoxia on pulmonary vasculature}

Hypoxia causes pulmonary hypertension in disease and at altitude. Evidence suggests that hypoxia-inducible factor (HIF) regulates the response to hypoxia across organ systems responsible for oxygen delivery to cells, including erythropoiesis, pulmonary and cardiovascular function. HIF is iron-dependent, requiring $\mathrm{Fe}(\mathrm{II})$ as a cofactor.

In two studies, the effect of iron infusion and depletion on pulmonary artery pressure (PASP) was investigated at altitude. In the first, healthy men resident at sea level were studied for 1 week in Cera de Pasco, Peru (altitude $4340 \mathrm{~m}$ ). During the first 3 days there were significant incremental increases in PASP. On day 3, participants received $200 \mathrm{mg}$ iron sucrose or placebo. The iron reversed much of the increase in PASP. A mean decrease of $6 \mathrm{~mm} \mathrm{Hg}$ was observed in the iron group compared with $2 \mathrm{~mm} \mathrm{Hg}$ in the placebo group.

In the second study the effect of iron depletion on chronic mountain sickness was investigated. The patients were iron depleted using staged venesection. There was a mean increase in PASP of $4 \mathrm{~mm} \mathrm{Hg}$ from $37 \mathrm{~mm} \mathrm{Hg}$ to $41 \mathrm{~mm} \mathrm{Hg}$. Over 2 weeks there was progressive iron deficiency, with a further rise in PASP. After 19 days the patients received $400 \mathrm{mg}$ iron sucrose or placebo followed by a crossover period. Following the initial infusions there was no difference between the groups, indicating that iron replacement did not acutely oppose the increase in PASP associated with iron depletion.

These studies suggest that iron may attenuate the response of the pulmonary vasculature to hypoxia and that iron deficiency may also be harmful in hypoxic pulmonary hypertension. This has implications for the management of chronic mountain sickness, where limiting red cell mass must be balanced against the risk of increasing PASP.

- Smith T, Talbot N, Privat C, et al. Effects of iron supplementation and depletion on hypoxic pulmonary hypertension: two randomized controlled trials. JAMA 2005;302:1444-50.

\section{Sarah Dyer}

Correspondence to: Sarah Dyer, ST4 in Respiratory Medicine, Wrexham Maelor Hospital, Wrexham, UK; sarah.dyer@doctors.net.uk

Thorax 2010:65:228. doi:10.1136/thx.2010.134825 